\title{
A NEW ALGORITHM OF PROPER GENERALIZED DECOMPOSITION FOR PARAMETRIC SYMMETRIC ELLIPTIC PROBLEMS*
}

\author{
M. AZAÏEZ ${ }^{\dagger}$, F. BEN BELGACEM ${ }^{\ddagger}$, J. CASADO-DÍAZ ${ }^{\S}$, T. CHACÓN REBOLLO『, AND \\ F. MURATII
}

\begin{abstract}
We introduce a new algorithm of proper generalized decomposition (PGD) for parametric symmetric elliptic partial differential equations. For any given dimension, we prove the existence of an optimal subspace of at most that dimension which realizes the best approximation-in the mean parametric norm associated to the elliptic operator - of the error between the exact solution and the Galerkin solution calculated on the subspace. This is analogous to the best approximation property of the proper orthogonal decomposition (POD) subspaces, except that in our case the norm is parameter-dependent. We apply a deflation technique to build a series of approximating solutions on finite-dimensional optimal subspaces, directly in the online step, and we prove that the partial sums converge to the continuous solution in the mean parametric elliptic norm. We show that the standard PGD for the considered parametric problem is strongly related to the deflation algorithm introduced in this paper. This opens the possibility of computing the PGD expansion by directly solving the optimization problems that yield the optimal subspaces.
\end{abstract}

Key words. proper orthogonal decomposition, proper generalized decomposition, online computation, reduced order modeling, symmetric elliptic problems

AMS subject classifications. 35J35, 35Pxx

DOI. $10.1137 / 17 \mathrm{M} 1137164$

1. Introduction. The Karhunen-Loève expansion (KLE) is a widely used tool that provides a reliable procedure for a low-dimensional representation of spatiotemporal signals (see [13, 23]). It is referred to as the principal components analysis (PCA) in statistics (see $[15,17,30]$ ) and called singular value decomposition (SVD) in linear algebra (see [14]). It is named proper orthogonal decomposition (POD) in mechanical computation, where it is also widely used (see [5]). Its use allows large savings of computational costs and makes affordable the solution of problems that need a large amount of solutions of parameter-dependent partial differential equations (PDEs); see $[4,10,16,21,30,31,32,34]$.

${ }^{*}$ Received by the editors July 5, 2017; accepted for publication (in revised form) July 9, 2018; published electronically October 11, 2018.

http://www.siam.org/journals/sima/50-5/M113716.html

Funding: The work of the first author was partially supported by the Spanish Government - Feder EU grant MTM2015-64577-C2-1-R and partially supported by NSFC/ANR joint program 51661135011 - PHASEFIELD. The work of the second author was partially supported by NSFC/ANR joint program 51661135011 - PHASEFIELD. The work of the fourth author was partially supported by the Spanish Government - Feder EU grant MTM2015-64577-C2-1-R. The work of the third and fifth authors was partially supported by the Spanish Government - Feder EU grant MTM2014-53309P.

${ }^{\dagger}$ Bordeaux INP, I2M (UMR CNRS 5295), Université de Bordeaux, 33607 Pessac, France, and EDAN \& IMUS, Universidad de Sevilla, 41012 Sevilla, Spain (Mejdi.Azaiez@enscbp.fr).

¥Sorbonne Universités, UTC, EA 2222, Laboratoire de Mathématiques Appliquées de Compiègne, 60205 Compiègne, France (fbenbelg@utc.fr).

$\S$ Departamento EDAN \& IMUS, Universidad de Sevilla, 41012 Sevilla, Spain (jcasadod@us.es).

ฯ I2M, IPB (UMR CNRS 5295), Université de Bordeaux, 33607 Pessac, France, and Departamento EDAN \& IMUS, Universidad de Sevilla, 41012 Sevilla, Spain (chacon@us.es).

"Laboratoire Jacques-Louis Lions, Boîte courrier 187, Sorbonne Universités, 75252 Paris cedex 05, France (murat@ann.jussieu.fr). 
However, the computation of the POD expansion requires knowledge of the function to be expanded, or at least its values at the nodes of a fine enough net. This makes it rather expensive to solve parametric elliptic PDEs, as it requires the previous solution of the PDE for a large enough number of values of the parameter ("snapshots"; see [18]), even if these can be located at optimal positions (see [20]). Galerkin-POD strategies are well suited to solving parabolic problems, where the POD basis is obtained from the previous solution of the underlying elliptic operator (see $[19,26])$.

An alternative approach is proper generalized decomposition (PGD) which iteratively computes a tensorized representation of the parameterized PDE that separates the parameter and the independent variables; this approach was introduced in [3]. It has been interpreted as a power type generalized spectral decomposition (see [27, 28]). It has experienced rapid development, being applied to the low-dimensional tensorized solution of many applied problems. The mathematical analysis of the PGD has experienced an important development in recent years. The convergence of a version of the PGD for symmetric elliptic PDEs via minimization of the associated energy has been proved in [22]. Also, in [11] the convergence of a recursive approximation of the solution of a linear elliptic PDE is proved, based on the existence of optimal subspaces of rank 1 that minimize the elliptic norm of the current residual.

The present paper is aimed at the direct determination of a variety of reduced dimension for the solution of parameterized symmetric elliptic PDEs. We intend to determine online an optimal subspace of given dimension that yields the best approximation in the mean (with respect to the parameter) of the error (in the parametric norm associated to the elliptic operator) between the exact solution and the Galerkin solution calculated on the subspace. The optimal POD subspaces can no longer be characterized by means of a spectral problem for a compact self-adjoint operator (the standard POD operator), and thus the spectral theory for compact self-adjoint operators does not apply. We build recursive approximations on finite-dimensional optimal subspaces by minimizing the mean parametric error of the current residual, similar to the one introduced in [11], that we prove to be strongly convergent in the "intrinsic" mean parametric elliptic norm. For this reason we call the method introduced the "intrinsic" PGD.

In addition, we prove that the method introduced is a genuine extension of both POD and PGD methods when applied to the solution of parametric elliptic equations. In particular it is strongly related to the PGD method in the sense that the standard formulation of the PGD method actually provides the optimality conditions of the minimization problem satisfied by the optimal 1D subspaces. As a consequence of the analysis developed in the paper, the PGD expansion is strongly convergent to the targeted solution in the parametric elliptic norm whenever it is implemented in such a way that all modes are optimal. Furthermore, the characterization of the modes by means of optimization problems opens the door to their computation by optimization techniques, in addition to the usual power iteration algorithm.

The abstract framework considered includes several kind of problems of practical interest, to which the PGD has been and continues to be applied. This is the case of the design analysis in computational mechanics. For instance, in the design of energy efficient devices (HVACs) or buildings, it is mandatory to address the heat equation with several structural parameters, for instance, the thermal diffusivity or transmittance, and the geometric shape of the device, among others. Also, the optimal design of heterogeneous materials that behave with linear law fits into the framework considered, as the parameters model the structural configuration of the various materials 
(cf. $[29,33])$. Moreover, in practice the structural configuration that optimizes a certain predefined criterion (e.g., construction costs, benefits, etc.) needs to take into account the unavoidable uncertainties in the structural performance. This leads to elliptic problems, including modeling of the targeted uncertainty, which, when the PDE model is linear, also fits into the abstract framework considered. In addition classical homogenization problems governed by linear symmetric elliptic PDEs also formally fit into this general framework, although the kind of approximation of the solution that is proposed in this work is different from the usual one, which looks for a limit averaged solution. Here we rather approximate the whole family of parameter-dependent solutions by a function series.

The method, however, does not apply, for instance, to nonsymmetric elliptic forms or to nonlinear problems.

The present paper focuses on theoretical aspects: We study the existence of the intrinsic POD and give a convergence result for the deflation algorithm. We defer the quantitative analysis of the convergence as well as numerical investigations to future work.

The paper is structured as follows: In section 2 we state the general problem of finding optimal subspaces of a given dimension. In section 3 we prove that there exists a solution for 1D optimal subspaces, characterized as a maximization problem with a nonlinear normalization restriction. We extend this existence result in section 4 to general dimensions. In section 5 we use the results from sections 3 and 4 to build a deflation algorithm to approximate the solution of a parametric family of elliptic problems, and we show the convergence. Section 6 explains why the method introduced is a genuine extension of both POD and PGD algorithms, and provides a theoretical analysis for the latter. Finally, in section 7 we present the main conclusions of the paper.

2. Statement of the problem. Let $H$ be a separable Hilbert space endowed with the scalar product $(\cdot, \cdot)$. The related norm is denoted by $\|\cdot\|$. We denote by $B_{s}(H)$ the space of bilinear, symmetric, and continuous forms in $H$.

Assume a given measure space $(\Gamma, \mathcal{B}, \mu)$, with standard notation, so that $\mu$ is $\sigma$-finite.

Let $a \in L^{\infty}\left(\Gamma, B_{s}(H) ; d \mu\right)$ be such that there exists $\alpha>0$ satisfying

$$
\alpha\|u\|^{2} \leq a(u, u ; \gamma) \quad \forall u \in H, d \mu \text {-a.e. } \gamma \in \Gamma .
$$

For $\mu$-a.e $\gamma \in \Gamma$, the bilinear form $a(\cdot, \cdot ; \gamma)$ determines a norm uniformly equivalent to the norm $\|\cdot\|$. Moreover, $\bar{a} \in B_{s}\left(L^{2}(\Gamma, H ; d \mu)\right)$ defined by

$$
\bar{a}(v, w)=\int_{\Gamma} a(v(\gamma), w(\gamma) ; \gamma) d \mu(\gamma) \quad \forall v, w \in L^{2}(\Gamma, H ; d \mu)
$$

defines an inner product in $L^{2}(\Gamma, H ; d \mu)$ which generates a norm equivalent to the standard one in $L^{2}(\Gamma, H ; d \mu)$.

Let there be given a data function $f \in L^{2}\left(\Gamma, H^{\prime} ; d \mu\right)$. We are interested in the following variational problem:

(3) Find $u(\gamma) \in H$ such that $a(u(\gamma), v ; \gamma)=\langle f(\gamma), v\rangle \quad \forall v \in H, d \mu$-a.e. $\gamma \in \Gamma$,

where $\langle\cdot, \cdot\rangle$ denotes the duality pairing between $H^{\prime}$ and $H$.

By the Riesz representation theorem, problem (3) admits a unique solution for $d \mu$-a.e. $\gamma \in \Gamma$. On the other hand, we claim that the $\tilde{u}$ solution of

$$
\tilde{u} \in L^{2}(\Gamma, H ; d \mu), \quad \bar{a}(\tilde{u}, \bar{v})=\int_{\Gamma}\langle f(\gamma), \bar{v}(\gamma)\rangle d \mu(\gamma) \quad \forall \bar{v} \in L^{2}(\Gamma, H ; d \mu)
$$


also satisfies (3): Indeed, taking $\bar{v}=v \chi_{B}$, with $v \in H$ fixed and $B \in \mathcal{B}$ arbitrary, implies that there exists a subset $N_{v} \in \mathcal{B}$ with $\mu\left(N_{v}\right)=0$ such that

$$
a(\tilde{u}(\gamma), v ; \gamma)=\langle f(\gamma), v\rangle \quad \forall \gamma \in \Gamma \backslash N_{v} .
$$

The separability of $H$ implies that $N_{v}$ can be chosen independent of $v$, which proves the claim. By the uniqueness of the solution of (3) this shows that

$$
\tilde{u}=u \quad d \mu \text {-a.e. } \gamma \in \Gamma \text {. }
$$

This proves that $u$ defined by (3) belongs to $L^{2}(\Gamma, H ; d \mu)$ and provides an equivalent definition of $u$, namely, that $u$ is the solution of (4).

Given a closed subspace $Z$ of $H$, let us denote by $u_{Z}(\gamma)$ the solution of the Galerkin approximation of problem (3) on $Z$, which is defined as

$$
u_{Z}(\gamma) \in Z, \quad a\left(u_{Z}(\gamma), z ; \gamma\right)=\langle f(\gamma), z\rangle \quad \forall z \in Z, d \mu \text {-a.e. } \gamma \in \Gamma
$$

or, equivalently, as

$$
u_{Z} \in L^{2}(\Gamma, Z ; d \mu), \quad \bar{a}\left(u_{Z}, z\right)=\int_{\Gamma}\langle f(\gamma), z(\gamma)\rangle d \mu(\gamma) \quad \forall z \in L^{2}(\Gamma, Z ; d \mu) .
$$

For every $k \in \mathbb{N}$, we intend to find the best subspace $W$ of $H$ of dimension less than or equal to $k$ that minimizes the mean error (in the norm defined by $\bar{a}$ ) between $u$ and $u_{W}$. That is, $W$ solves

$$
\min _{Z \in \mathbb{G} \leq k} \bar{a}\left(u-u_{Z}, u-u_{Z}\right),
$$

where $\mathbb{G}_{\leq k}$ is the family of subspaces of $H$ of dimension less than or equal to $k$. Note that $\mathbb{G}_{\leq k}$ is a connected component of the Grassmaniann variety $\mathbb{G}_{\leq k}$ of $H$, defined as

$$
\mathbb{G}_{\leq k}=\bigcup_{k \geq 0} \mathbb{G}_{k}
$$

where $\mathbb{G}_{k}$ is the set formed by all subspaces of $H$ of dimension $k$. The set $\mathbb{G}_{k}$ is a Hilbert manifold modeled in a particular Hilbert space (see [1, 25]).

Problem (8) will be proved to have a solution in sections 3 and 4 . We will then use this result in section 5 to approximate the solution $u$ of problem (3) by a deflation algorithm.

Note that when looking at the formulation of the minimization problem (8), it seems that solving it requires the knowledge of the solution $u$ of (3). But such is not the case, since Proposition 2.6 below provides an equivalent formulation of (8) which does not depend on the knowledge of $u$ but only on the data $f$.

Let us provide some equivalent formulations of problem (8). First we observe the following.

Proposition 2.1. For every closed subspace $Z \subset H$, the function $u_{Z}$ defined by (7) is also the unique solution of

$$
\min _{z \in L^{2}(\Gamma, Z ; d \mu)} \bar{a}(u-z, u-z) .
$$

Moreover, for $d \mu$-a.e. $\gamma \in \Gamma$, the vector $u_{Z}(\gamma)$ is the solution of

$$
\min _{z \in Z} a(u(\gamma)-z, u(\gamma)-z ; \gamma)
$$

Copyright (C) by SIAM. Unauthorized reproduction of this article is prohibited. 
Proof. It is a classical property of the Galerkin approximation of the variational formulation of linear elliptic problems that $u_{Z}$ satisfies (9). Indeed, the symmetry of $\bar{a}$ gives

$$
\bar{a}(u-z, u-z)=\bar{a}\left(u-u_{Z}, u-u_{Z}\right)+2 \bar{a}\left(u-u_{Z}, u_{Z}-z\right)+\bar{a}\left(u_{Z}-z, u_{Z}-z\right)
$$

for every $z \in L^{2}(\Gamma, H ; d \mu)$, where by (4), (5), and (7) the second term on the righthand side vanishes, while the third one is nonnegative. This proves (9).

The proof of (10) is the same by taking into account (3) and (6) instead of (4) and (7).

As a consequence of Proposition 2.1 and definition (2) of $\bar{a}$, we have the following corollary.

Corollary 2.2. A space $W \in \mathbb{G}_{\leq k}$ is a solution of (8) if and only if it is a solution of

$$
\min _{Z \in \mathbb{G} \leq k} \min _{z \in L^{2}(\Gamma, Z ; d \mu)} \bar{a}(u-z, u-z)
$$

Moreover,

$$
\min _{Z \in \mathbb{G} \leq k} \min _{z \in L^{2}(\Gamma, Z ; d \mu)} \bar{a}(u-z, u-z)=\min _{Z \in \mathbb{G} \leq k} \int_{\Gamma} \min _{z \in Z} a(u(\gamma)-z, u(\gamma)-z ; \gamma) d \mu(\gamma)
$$

Remark 2.3. Optimization problem (11) is reminiscent of the Kolmogorov $k$ width related to the best approximation of the manifold $(u(\gamma))_{\gamma \in \Gamma}$ by subspaces in $H$ with dimension $k$ as presented in [24]. In the present minimization problem, we use the norm of $L^{2}(\Gamma, H ; d \mu)$ instead of the norm of $L^{\infty}(\Gamma, H ; d \mu)$ as used there. The minimization problem in [24] can indeed be written as

$$
\min _{Z \in \mathbb{G} \leq k} \operatorname{esssup} \min _{z \in \Gamma} a(u(\gamma)-z, u(\gamma)-z ; \gamma)
$$

if one uses $a(\cdot, \cdot ; \gamma)$ as the inner product in $H$.

The analysis performed in the present paper is strongly based on the Hilbertian framework associated to the minimization in $L^{2}(\Gamma, H ; d \mu)$. To the best of our knowledge, few facts are known about problem (13); in particular, there is no proof of existence of solutions. The extension to this problem of the techniques used in the present paper is far from being straightforward, and we intend to discuss this in a future paper. Indeed, the $L^{\infty}(\Gamma, H ; d \mu)$ framework is especially interesting whenever uniform error estimates with respect to the parameter are needed. This happens, for instance, when upper bounds for energy consumption (mechanical, thermal, etc.) should be respected.

For a function $v \in L^{2}(\Gamma, H ; d \mu)$, we denote by $R(v)$ the closure of the vectorial space spanned by $v(\gamma)$ when $\gamma$ belongs to $\Gamma$; more exactly, taking into account that $v$ is only defined up to sets of zero measure, the correct definition of $R(v)$ is given by

$$
R(v)=\bigcap_{\mu(N)=0} \overline{\operatorname{Span}\{v(\gamma): \gamma \in \Gamma \backslash N\}}
$$

The following result proves that in (14) the intersection can be replaced by a single closed spanned space corresponding to a single set $M \in \mathcal{B}$. This proves in particular that it does not reduce to $\{0\}$ if $R(v)$ is not zero $d \mu$-a.e. $\gamma \in \Gamma$. 
Proposition 2.4. For every $v \in L^{2}(\Gamma, H ; d \mu)$ there exists $M \in \mathcal{B}$, with $\mu(M)=0$ such that

$$
R(v)=\overline{\operatorname{Span}\{v(\gamma): \gamma \in \Gamma \backslash M\}} .
$$

Proof. For every $N \in \mathcal{B}$, we define $P_{N}$ as the orthogonal projection of $H$ into

$$
R_{N}:=\overline{\operatorname{Span}\{v(\gamma): \gamma \in \Gamma \backslash N\}} .
$$

We also define $P$ as the orthogonal projection of $H$ into $R(v)$.

Let us first prove that

$$
\forall z \in H, \exists M_{z} \in \mathcal{B} \text { with } \mu\left(M_{z}\right)=0 \text { such that } P z=P_{M_{z}} z .
$$

In order to prove this result, we consider $N_{n} \in \mathcal{B}$, with $\mu\left(N_{n}\right)=0$, such that

$$
\left\|P_{N_{n}} z\right\| \rightarrow \inf _{\mu(N)=0}\left\|P_{N} z\right\| .
$$

Taking $M_{z}=\cup_{n} N_{n}$, we have that $\mu\left(M_{z}\right)=0$. Moreover, using that $N_{n} \subset M_{z}$ implies $R_{M_{z}} \subset R_{N_{n}}$, we get

$$
\inf _{\mu(N)=0}\left\|P_{N} z\right\| \leq\left\|P_{M_{z}} z\right\| \leq\left\|P_{N_{n}} z\right\| \quad \forall n \geq 1 .
$$

Therefore,

$$
\left\|P_{M_{z}} z\right\|=\inf _{\mu(N)=0}\left\|P_{N} z\right\| .
$$

Now, we use that for every $N \in \mathcal{B}$ with $M_{z} \subset N, \mu(N)=0$, we have

$$
R_{N} \subset R_{M_{z}}, \quad\left\|P_{M_{z}} z\right\| \leq\left\|P_{N} z\right\|,
$$

and then

$$
P_{N} z=P_{M_{z}} z \quad \forall N \supset M_{z} \text { with } \mu(N)=0 .
$$

We take now an arbitrary $N \in \mathcal{B}$ with $\mu(N)=0$. Using $M_{z} \subset N \cup M_{z}, \mu\left(N \cup M_{z}\right)=0$, and (16), we get

$$
P_{M_{z}} z=P_{N \cup M_{z}} z \in R_{N \cup M_{z}} \subset R_{N} \quad \forall N \in \mathcal{B} \text {, with } \mu(N)=0,
$$

and so $P_{M_{z}} z$ belongs to $R(v)$. On the other hand, observe that $R(v) \subset R_{M_{z}}$ and the definition of $P_{M_{z}}$ imply

$$
\left\|z-P_{M_{z}} z\right\| \leq\|z-\eta\| \quad \forall \eta \in R(v)
$$

and thus $P_{M_{z}} z=P z$. This proves (15).

Let us now use (15) to prove the statement of Proposition 2.4. We consider an orthonormal basis $\left\{z_{k}\right\}$ of $R(v)^{\perp}$. By (15), we know that for every $k \geq 1$, there exists $M_{z_{k}} \in \mathcal{B}$ with $\mu\left(M_{z_{k}}\right)=0$ such that $P_{M_{k}} z_{k}=0$. Then we define

$$
M=\bigcup_{k \geq 1} M_{z_{k}} .
$$

Let us prove that $M$ satisfies the thesis of the proposition. Clearly $\mu(M)=0$; moreover, (16) and $M_{z_{k}} \subset M$ for every $k \geq 1$ imply

$$
P_{M} e_{k}=0 \quad \forall k \geq 1 \text {. }
$$

This shows $P_{M} z=0$ for every $z \in R(v)^{\perp}$, and then $R(v)^{\perp} \subset R_{M}^{\perp}$ or, equivalently, $R_{M} \subset R(v)$. Since the other contention is immediate, we have then proved $R_{M}=$ $R(v)$, which finishes the proof.

Copyright $@$ by SIAM. Unauthorized reproduction of this article is prohibited. 
Taking into account (11), a new formulation of (8) is given by the following.

Proposition 2.5. If $W$ is a solution of (8), then $u_{W}$ is a solution of

$$
\min _{\substack{v \in L^{2}(\Gamma, H ; d \mu) \\ \operatorname{dim} R(v) \leq k}} \bar{a}(u-v, u-v) .
$$

Reciprocally, if $\hat{u}$ is a solution of (17), then $R(\hat{u})$ is a solution of (8) and $\hat{u}=u_{R(\hat{u})}$.

As announced above, the next proposition provides an equivalent formulation for (8) which does not depend on the knowledge of the solution u of (3) but only on the data $f$.

Proposition 2.6. The subspace $W \in \mathbb{G}_{\leq k}$ solves problem (8) if and only if it is a solution of the problem

$$
\max _{Z \in \mathbb{G}_{\leq k}} \int_{\Gamma}\left\langle f(\gamma), u_{Z}(\gamma)\right\rangle d \mu(\gamma),
$$

where $u_{Z}$ is defined by (7).

Proof. As in the proof of the first part of Proposition 2.1, one deduces from (4), (5), and (7) that

$$
\bar{a}\left(u-u_{Z}, z\right)=0 \quad \forall z \in L^{2}(\Gamma, Z ; d \mu) .
$$

Using the symmetry of $\bar{a}$, we then have

$$
\begin{aligned}
& \bar{a}\left(u-u_{Z}, u-u_{Z}\right)=\bar{a}(u, u)-a\left(u_{Z}, u\right)=\bar{a}(u, u)-\bar{a}\left(u_{Z}, u_{Z}\right) \\
& =\bar{a}(u, u)-\int_{\Gamma}\left\langle f(\gamma), u_{Z}(\gamma)\right\rangle d \mu(\gamma) .
\end{aligned}
$$

Thus $W$ solves (8) if and only if it solves (18).

3. One-dimensional approximations. In section 4 we shall show the existence of the solution of problem (8) for any arbitrary $k$. However a particularly interesting case from the point of view of the applications is $k=1$. We dedicate this section to this special case. Observe that for $Z \in \mathbb{G}_{1}$, there exists $z \in H \backslash\{0\}$ such that $Z=\operatorname{Span}\{z\}$. The problem to solve can be reformulated as follows.

Lemma 3.1. Assume $f \not \equiv 0$. Then, the subspace $W \in \mathbb{G}_{1}$ solves problem (18) if and only if $W=\operatorname{Span}\{w\}$, where $w$ is a solution of

$$
\max _{\substack{z \in H \\ z \neq 0}} \int_{\Gamma} \frac{\langle f(\gamma), z\rangle^{2}}{a(z, z ; \gamma)} d \mu(\gamma)
$$

Proof. Let $Z \in \mathbb{G}_{1}$. Then $Z=\operatorname{Span}\{z\}$, for some $z \in H \backslash\{0\}$, and there exists a function $\varphi: \Gamma \mapsto \mathbb{R}$ such that

$$
u_{Z}(\gamma)=\varphi(\gamma) z, \quad d \mu \text {-a.e. } \gamma \in \Gamma .
$$

As $z \neq 0$, then, as $u_{Z}(\gamma)$ is the solution to the variational equation (6), we derive that

$$
\varphi(\gamma)=\frac{\langle f(\gamma), z\rangle}{a(z, z ; \gamma)}, \quad d \mu \text {-a.e. } \gamma \in \Gamma .
$$

Using this formula we obtain that

$$
\int_{\Gamma}\left\langle f, u_{Z}(\gamma)\right\rangle d \gamma=\int_{\Gamma} \frac{\langle f(\gamma), z\rangle^{2}}{a(z, z ; \gamma)} d \mu(\gamma)
$$

Copyright $@$ by SIAM. Unauthorized reproduction of this article is prohibited. 
If the maximum in (18) is obtained by a space of dimension one, then formula (20) proves the desired result.

In contrast, if the maximum in (18) is obtained by the null space, then the maximum in $\mathbb{G}_{1}$ is equal to zero. Therefore the right-hand side of $(20)$ is zero for every $z \in H$, which implies that $f=0 d \mu$-a.e. in $\Gamma$, in contradiction with the assumption $f \not \equiv 0$.

Remark 3.2. Since the integrand which appears in (19) is homogeneous of degree zero in $z$, problem (19) is equivalent to

$$
\max _{\substack{z \in H \\\|z\|=1}} \int_{\Gamma} \frac{\langle f(\gamma), z\rangle^{2}}{a(z, z ; \gamma)} d \mu(\gamma)
$$

We now prove the existence of a solution to problem (19).

Theorem 3.3. Assume $f \not \equiv 0$. Problem (19) admits at least one solution.

Note that if $f \equiv 0$, then every vector $w \in H \backslash\{0\}$ is a solution of (19).

Proof. Define

$$
M^{*}:=\sup _{\substack{z \in H \\\|z\|=1}} \int_{\Gamma} \frac{\langle f(\gamma), z\rangle^{2}}{a(z, z ; \gamma)} d \mu(\gamma),
$$

and consider a sequence $w_{n} \subset H$, with $\left\|w_{n}\right\|=1$ such that

$$
\lim _{n \rightarrow \infty} \int_{\Gamma} \frac{\left\langle f(\gamma), w_{n}\right\rangle^{2}}{a\left(w_{n}, w_{n} ; \gamma\right)} d \mu(\gamma)=M^{*}
$$

Up to a subsequence, we can assume the existence of $w \in H$, such that $w_{n}$ converges weakly in $H$ to $w$. Taking into account that $f(\gamma) \in H^{\prime}, a(\cdot, \cdot, \gamma) \in B_{s}(H) d \mu$-a.e. $\gamma \in \Gamma$, and (1) is satisfied, we get

$$
\begin{gathered}
\lim _{n \rightarrow \infty}\left\langle f(\gamma), w_{n}\right\rangle=\langle f(\gamma), w\rangle, \quad d \mu \text {-a.e. } \gamma \in \Gamma, \\
\liminf _{n \rightarrow \infty} a\left(w_{n}, w_{n} ; \gamma\right) \geq a(w, w ; \gamma), \quad d \mu \text {-a.e. } \gamma \in \Gamma .
\end{gathered}
$$

On the other hand, we observe that (1) and $\left\|w_{n}\right\|=1$ imply

$$
\left|\left\langle f(\gamma), w_{n}\right\rangle\right| \leq\|f(\gamma)\|_{H^{\prime}}, \quad \frac{1}{a\left(w_{n}, w_{n} ; \gamma\right)} \leq \frac{1}{\alpha}, \quad d \mu \text {-a.e. } \gamma \in \Gamma .
$$

If $w=0$, then (23), (25), and Lebesgue's dominated convergence theorem imply

$$
\lim _{n \rightarrow \infty} \int_{\Gamma} \frac{\left\langle f(\gamma), w_{n}\right\rangle^{2}}{a\left(w_{n}, w_{n} ; \gamma\right)} d \mu(\gamma)=0
$$

which by (22) is equivalent to $M^{*}=0$. Taking into account (1) and the definition (21) of $M^{*}$, this is only possible if $f \equiv 0$ is the null function. As we are assuming $f \not \equiv 0$, we conclude that $w$ is different from zero. Then, (25) proves

$$
0 \leq \frac{\|f(\gamma)\|_{H^{\prime}}^{2}}{\alpha}-\frac{\left\langle f(\gamma), w_{n}\right\rangle^{2}}{a\left(w_{n}, w_{n} ; \gamma\right)}, \quad d \mu \text {-a.e. } \gamma \in \Gamma,
$$


while (23) and (24) prove

$$
\liminf _{n \rightarrow \infty}\left(\frac{\|f(\gamma)\|_{H^{\prime}}^{2}}{\alpha}-\frac{\left\langle f(\gamma), w_{n}\right\rangle^{2}}{a\left(w_{n}, w_{n} ; \gamma\right)}\right) \geq \frac{\|f(\gamma)\|_{H^{\prime}}^{2}}{\alpha}-\frac{\langle f(\gamma), w\rangle^{2}}{a(w, w ; \gamma)}, \quad d \mu \text {-a.e. } \gamma \in \Gamma .
$$

Using (22), Fatou's lemma (see [6, section 4.1]) implies

$$
\begin{aligned}
& \int_{\Gamma}\left(\frac{\|f(\gamma)\|_{H^{\prime}}^{2}}{\alpha}-\frac{\langle f(\gamma), w\rangle^{2}}{a(w, w ; \gamma)}\right) d \mu(\gamma) \leq \liminf _{n \rightarrow \infty} \int_{\Gamma}\left(\frac{\|f(\gamma)\|_{H^{\prime}}^{2}}{\alpha}-\frac{\left\langle f(\gamma), w_{n}\right\rangle^{2}}{a\left(w_{n}, w_{n} ; \gamma\right)}\right) d \mu(\gamma) \\
& =\int_{\Gamma} \frac{\|f(\gamma)\|_{H^{\prime}}^{2}}{\alpha} d \mu(\gamma)-M^{*}
\end{aligned}
$$

or, equivalently,

$$
M^{*} \leq \int_{\Gamma} \frac{\langle f(\gamma), w\rangle^{2}}{a(w, w ; \gamma)} d \mu(\gamma)
$$

By definition (21) of $M^{*}$, this proves that the above inequality is an equality and that $w$ is a solution of (19).

Remark 3.4. Actually, in place of (26), one has the stronger result

$\liminf _{n \rightarrow \infty}\left(\frac{\|f(\gamma)\|_{H^{\prime}}^{2}}{\alpha}-\frac{\left\langle f(\gamma), w_{n}\right\rangle^{2}}{a\left(w_{n}, w_{n} ; \gamma\right)}\right)=\frac{\|f(\gamma)\|_{H^{\prime}}^{2}}{\alpha}-\frac{\langle f(\gamma), w\rangle^{2}}{\liminf _{n \rightarrow \infty} a\left(w_{n}, w_{n} ; \gamma\right)}, \quad d \mu$-a.e. $\gamma \in \Gamma$,

which by the proof used to prove (27) shows

$$
M^{*} \leq \int_{\Gamma} \frac{\langle f(\gamma), w\rangle^{2}}{\liminf _{n \rightarrow \infty} a\left(w_{n}, w_{n} ; \gamma\right)} d \mu(\gamma) .
$$

Combined with

$$
M^{*}=\int_{\Gamma} \frac{\langle f(\gamma), w\rangle^{2}}{a(w, w ; \gamma)} d \mu(\gamma)
$$

and (24), this implies

$$
a(w, w ; \gamma)=\liminf _{n \rightarrow \infty} a\left(w_{n}, w_{n} ; \gamma\right) \quad d \mu \text {-a.e. } \gamma \in \Gamma \text { such that }\langle f(\gamma), w\rangle \neq 0
$$

By (1) and $f \not \equiv 0$, this proves the existence of a subsequence of $w_{n}$ which converges strongly to $w$ a.e. $\gamma$.

Since this proof can be carried out by replacing $w_{n}$ with any subsequence of $w_{n}$, we conclude that the whole sequence $w_{n}$ (which we extracted just after (22), assuming that it converges weakly to some $w$ ) actually converges strongly to $w$.

The above result may be used to build a computable approximation of a solution of (19). Indeed, for $f \not \equiv 0$, let $\left\{H_{n}\right\}_{n \geq 1}$ be an internal approximation of $H$, that is, a sequence of subspaces of finite dimension of $H$ such that

$$
\lim _{n \rightarrow \infty} \inf _{\psi \in H_{n}}\|z-\psi\|=0 \quad \forall z \in H
$$

and consider a solution $w_{n}$ of

$$
\max _{\substack{z \in H_{n} \\\|z\|=1}} \int_{\Gamma} \frac{\langle f(\gamma), z\rangle^{2}}{a(z, z ; \gamma)} d \mu(\gamma) .
$$

Copyright $@$ by SIAM. Unauthorized reproduction of this article is prohibited. 
The existence of such a $w_{n}$ can be obtained by the same reasoning as in the proof of Theorem 3.3 or by just using the Weierstrass theorem because the dimension of $H_{n}$ is finite.

Taking $\tilde{w}$ a solution of (19) and a sequence $\tilde{w}_{n} \in H_{n}$ converging to $\tilde{w}$ in $H$, we have

$$
\begin{aligned}
& \int_{\Gamma} \frac{\langle f(\gamma), \tilde{w}\rangle^{2}}{a(\tilde{w}, \tilde{w} ; \gamma)} d \mu(\gamma)=\lim _{n \rightarrow \infty} \int_{\Gamma} \frac{\left\langle f(\gamma), \tilde{w}_{n}\right\rangle^{2}}{a\left(\tilde{w}_{n}, \tilde{w}_{n} ; \gamma\right)} d \mu(\gamma) \\
& \leq \liminf _{n \rightarrow \infty} \int_{\Gamma} \frac{\left\langle f(\gamma), w_{n}\right\rangle^{2}}{a\left(w_{n}, w_{n} ; \gamma\right)} d \mu(\gamma) \leq \limsup _{n \rightarrow \infty} \int_{\Gamma} \frac{\left\langle f(\gamma), w_{n}\right\rangle^{2}}{a\left(w_{n}, w_{n} ; \gamma\right)} d \mu(\gamma) \leq \int_{\Gamma} \frac{\langle f(\gamma), \tilde{w}\rangle^{2}}{a(\tilde{w}, \tilde{w} ; \gamma)} d \mu(\gamma),
\end{aligned}
$$

and thus

$$
\lim _{n \rightarrow \infty} \int_{\Gamma} \frac{\left\langle f(\gamma), w_{n}\right\rangle^{2}}{a\left(w_{n}, w_{n} ; \gamma\right)} d \mu(\gamma)=\int_{\Gamma} \frac{\langle f(\gamma), \tilde{w}\rangle^{2}}{a(\tilde{w}, \tilde{w} ; \gamma)} d \mu(\gamma)=M^{*}
$$

This proves that the sequence $w_{n}$ satisfies (22). Therefore any subsequence of $w_{n}$ that converges weakly to some $w$ converges strongly to $w$ which is a solution of (19).

4. Higher-dimensional approximations. This section is devoted to the proof of the existence of an optimal subspace which is a solution of (8) when $k \geq 1$ is any given number.

TheOREm 4.1. For any given $k \geq 1$, problem (8) admits at least one solution.

Proof. As in the proof of Theorem 3.3, we use the direct method of the calculus of variations. Denoting by $m_{k}$

$$
m_{k}=\inf _{Z \in \mathbb{G}_{\leq k}} \bar{a}\left(u-u_{Z}, u-u_{Z}\right),
$$

we consider a sequence of spaces $W_{n} \in \mathbb{G}_{\leq k}$ such that $w_{n}:=u_{W_{n}}$ satisfies

$$
\lim _{n \rightarrow \infty} \bar{a}\left(u-w_{n}, u-w_{n}\right)=m_{k} .
$$

Taking into account that by Proposition 2.1

$$
Z \subset \tilde{Z} \Longrightarrow \bar{a}\left(u-u_{\tilde{Z}}, u-u_{\tilde{Z}}\right) \leq \bar{a}\left(u-u_{Z}, u-u_{Z}\right),
$$

we can assume that the dimension of $W_{n}$ is equal to $k$. Moreover, we observe that (29) implies that $w_{n}$ is bounded in $L^{2}(\Gamma, H ; d \mu)$.

Let $\left(z_{n}^{1}, \ldots, z_{n}^{k}\right)$ be an orthonormal basis of $W_{n}$. It holds that

$$
w_{n}(\gamma)=\sum_{j=1}^{k}\left(w_{n}(\gamma), z_{n}^{j}\right) z_{n}^{j}, \quad d \mu \text {-a.e. } \gamma \in \Gamma .
$$

Since the norm of the vectors $z_{n}^{j}$ is one, there exists a subsequence of $n$ and $k$ vectors $z^{j} \in H$ such that

$$
z_{n}^{j} \rightarrow z^{j} \text { in } H \quad \forall j \in\{1, \ldots, k\} .
$$

Using also

$$
\left|\left(w_{n}(\gamma), z_{n}^{j}\right)\right| \leq\left\|w_{n}(\gamma)\right\|, \quad d \mu \text {-a.e } \gamma \in \Gamma,
$$

we get that $\left(w_{n}, z_{n}^{j}\right)$ is bounded in $L^{2}(\Gamma, H ; d \mu)$ for every $j$, and thus there exists a subsequence of $n$ and $k$ functions $p^{j} \in L^{2}(\Gamma ; d \mu)$ such that

$$
\left(w_{n}, z_{n}^{j}\right) \rightarrow p^{j} \quad \text { in } L^{2}(\Gamma, H ; d \mu) \quad \forall j \in\{1, \ldots, k\} .
$$


We claim that

$$
w_{n} \rightarrow w:=\sum_{j=1}^{n} p^{j} z^{j} \quad \text { in } L^{2}(\Gamma ; d \mu)
$$

Indeed, taking into account that $w_{n}$ is bounded in $L^{2}(\Gamma, H ; d \mu)$ and (31), it is enough to show

$$
\lim _{n \rightarrow \infty} \int_{\Gamma}\left(\left(w_{n}, z_{n}^{j}\right) z_{n}^{j}, \varphi v\right) d \mu(\gamma)=\int_{\Gamma}\left(p^{j} z^{j}, \varphi v\right) d \mu(\gamma) \quad \forall \varphi \in L^{2}(\Gamma ; d \mu), \forall v \in H .
$$

This is a simple consequence of

$$
\int_{\Gamma}\left(\left(w_{n}, z_{n}^{j}\right) z_{n}^{j}, \varphi v\right) d \mu(\gamma)=\left(z_{n}^{j}, v\right) \int_{\Gamma}\left(w_{n}, z_{n}^{j}\right) \varphi d \mu(\gamma),
$$

combined with (32) and (33).

From the continuity and convexity of the quadratic form associated to $\bar{a}$, as well as from (34) and (29), we have

$$
\bar{a}(u-w, u-w) \leq \lim _{n \rightarrow \infty} \bar{a}\left(u-w_{n}, u-w_{n}\right)=m_{k} .
$$

Using that $W=\operatorname{Span}\left\{z^{1}, \ldots, z^{k}\right\} \in \mathbb{G}_{\leq k}$, and that (see Proposition 2.1)

$$
\bar{a}\left(u-u_{W}, u-u_{W}\right) \leq \bar{a}(u-w, u-w),
$$

we conclude that $W$ is a solution of $(8)$.

Remark 4.2. From (36), (37), definition (28) of $m_{k}$, and Proposition 2.1, we have that $w=u_{W}$ in the proof of Theorem 4.1. Moreover,

$$
\bar{a}(u-w, u-w)=m_{k}=\lim _{n \rightarrow \infty} \bar{a}\left(u-w_{n}, u-w_{n}\right),
$$

which combined with (34) proves that $w_{n}$ converges strongly to $w$ in $L^{2}(\Gamma, H ; d \mu)$. As in Remark 3.4, this can be used to build a strong approximation of a solution of (8) by using an internal approximation of $H$.

5. An iterative algorithm by deflation. In the previous section, for any given $k \geq 1$, we have proved the existence of an optimal subspace for problem (8). We use this fact here to build an iterative approximation of the solution of (3) by a deflation approach. We build recursive approximations on finite-dimensional optimal subspaces by minimizing the mean parametric error of the current residual, similar to the recursive approximations introduced in [11]. Let us denote

$$
\Pi_{k}(v)=\left\{v_{W} \mid \quad W \text { solves } \min _{Z \in \mathbb{G}_{\leq k}} \bar{a}\left(v-v_{Z}, v-v_{Z}\right),\right\} \quad \forall v \in L^{2}(\Gamma, H ; d \mu)
$$

where $v_{Z}$ is defined by $(7)$. 
The deflation algorithm is as follows:

- Initialization:

$$
u_{0}=0
$$

- Iteration: Assuming $u_{i-1} \in H$ known for $i=1,2, \ldots$, set

(40) $e_{i-1}=u-u_{i-1}$, choose $s_{i} \in \Pi_{k}\left(e_{i-1}\right)$, and define $u_{i}=u_{i-1}+s_{i}$.

Remark 5.1. Note that $s_{i}$ (and therefore $u_{i}$ ) in general is not defined in a unique way.

Note also that algorithm (40) does not need to know the solution $u$ of (4), since $e_{i-1}=u-u_{i-1}$ is directly defined from $f$ and $u_{i-1}$ by

$$
\left\{\begin{array}{l}
e_{i-1} \in L^{2}(\Gamma, H ; d \mu), \\
\bar{a}\left(e_{i-1}, v\right)=\int_{\Gamma}\langle f(\gamma), v(\gamma)\rangle d \mu(\gamma)-\bar{a}\left(u_{i-1}, v\right) \quad \forall v \in L^{2}(\Gamma, H ; d \mu) .
\end{array}\right.
$$

Then Proposition 2.6, applied to the case where $f$ is replaced by the function $f_{i}$ defined by

$$
\int_{\Gamma}\left\langle f_{i}(\gamma), v(\gamma)\right\rangle d \mu(\gamma)=\int_{\Gamma}\langle f(\gamma), v(\gamma)\rangle d \mu(\gamma)-\bar{a}\left(u_{i-1}, v\right) \quad \forall v \in L^{2}(\Gamma, H ; d \mu),
$$

proves that

$$
s_{i} \in \Pi_{k}\left(e_{i-1}\right) \Longleftrightarrow s_{i}=\left(e_{i-1}\right)_{W_{i}},
$$

where $W_{i}$ is a solution of

$$
\max _{Z \in \mathbb{G}_{\leq k}}\left\{\int_{\Gamma}\left\langle f(\gamma),\left(e_{i-1}\right)_{Z}(\gamma)\right\rangle d \mu(\gamma)-\bar{a}\left(u_{i-1},\left(e_{i-1}\right)_{Z}\right)\right\},
$$

where, in accordance with $(7),\left(e_{i-1}\right)_{Z}$ denotes the solution of

$$
\left\{\begin{array}{l}
\left(e_{i-1}\right)_{Z} \in L^{2}(\Gamma, Z ; d \mu), \\
\bar{a}\left(\left(e_{i-1}\right)_{Z}, z\right)=\int_{\Gamma}\langle f(\gamma), z(\gamma)\rangle d \mu(\gamma)-\bar{a}\left(u_{i-1}, z\right) \quad \forall z \in L^{2}(\Gamma, Z ; d \mu) .
\end{array}\right.
$$

This observation allows one to carry out the iterative process without knowing the function $u$.

Note also that

$$
u_{i}=\sum_{j=1}^{i} s_{j}
$$

namely that $u_{i}$ is the partial sum of the series $\sum_{j \geq 1} s_{j}$.

Remark 5.2. In this remark we take $k=1$. Then every space of $\mathbb{G}_{\leq 1}$ is spanned by an element of $H$, and in particular $W_{i}=\operatorname{Span}\left\{w_{i}\right\}$ for some $w_{i} \in H$; then $u_{i}(\gamma)=\sum_{j=1}^{i} \Phi_{j}(\gamma) w_{j}$, where $\Phi_{j}(\gamma) \in L^{2}(\Gamma, d \mu)$ is defined by $s_{j}=\Phi_{j}(\gamma) w_{j}$. Note also that if $w_{i}=0$ for some $i \geq 0$, then $f_{i}=0$ and thus $u \equiv u_{i-1}$,

The convergence of the algorithm is given by the following theorem. Its proof follows the ideas of [11].

Copyright $@$ by SIAM. Unauthorized reproduction of this article is prohibited. 
THEOREM 5.3. The sequence $u_{i}$ provided by the least-squares PGD algorithm (39)-(40) strongly converges in $L^{2}(\Gamma, H ; d \mu)$ to the parameterized solution $\gamma \in \Gamma \mapsto$ $u(\gamma) \in H$ of problem $(3)$.

Remark 5.4. In view of the last assertion of Remark 5.1, Theorem 5.3 proves that the series $\sum_{j \geq 1} s_{j}$ converges in $L^{2}(\Gamma, H ; d \mu)$ to the parameterized solution $\gamma \in \Gamma \mapsto$ $u(\gamma) \in H$ of problem $(3)$.

When $k=1$, Remark 5.2 implies that the series $\sum_{j \geq 1} \Phi_{j}(\gamma) w_{j}$ converges in $L^{2}(\Gamma, H ; d \mu)$ to this parameterized solution.

Proof. By (40) and Proposition 2.5 applied to the case where $u$ is replaced by $e_{i-1}$, we have that $s_{i}$ is a solution of

$$
\min _{\substack{v \in L^{2}(\Gamma, H ; d \mu) \\ \operatorname{dim} R(v) \leq k}} \bar{a}\left(e_{i-1}-v, e_{i-1}-v\right)
$$

This proves in particular that $s_{i}$ is a solution of

$$
\min _{\substack{v \in L^{2}(\Gamma, H ; d \mu) \\ R(v) \subset R\left(s_{i}\right)}} \bar{a}\left(e_{i-1}-v, e_{i-1}-v\right),
$$

and therefore,

$$
\bar{a}\left(e_{i-1}-s_{i}, v\right)=0 \quad \forall v \in L^{2}(\Gamma, H ; d \mu) \text { with } R(v) \subset R\left(s_{i}\right) .
$$

But (40) implies that

$$
e_{i-1}-s_{i}=e_{i}
$$

which gives

$$
\bar{a}\left(e_{i}, v\right)=0 \quad \forall v \in L^{2}(\Gamma, H ; d \mu) \text { with } R(v) \subset R\left(s_{i}\right) .
$$

Taking $v=s_{i}$ and using again (46) we get

$$
\bar{a}\left(e_{i-1}, e_{i-1}\right)=\bar{a}\left(s_{i}, s_{i}\right)+\bar{a}\left(e_{i}, e_{i}\right) \quad \forall i \geq 1,
$$

and therefore,

$$
\bar{a}\left(e_{i}, e_{i}\right)+\sum_{j=1}^{i} \bar{a}\left(s_{j}, s_{j}\right)=\bar{a}\left(e_{0}, e_{0}\right) \quad \forall i \geq 1
$$

Thus, we have that

$$
e_{i} \text { is bounded in } L^{2}(\Gamma, H ; d \mu) \text {, }
$$

By (50), there exists a subsequence $e_{i_{n}}$ of $e_{i}$ and $e \in L^{2}(\Gamma, H ; d \mu)$, such that

$$
e_{i_{n}} \rightarrow e \text { in } L^{2}(\Gamma, H ; d \mu)
$$

Copyright (C) by SIAM. Unauthorized reproduction of this article is prohibited. 
On the other hand, since $s_{i_{n}+1}$ is a solution of (45) with $i-1$ replaced by $i_{n}$, we get (53)

$$
\begin{aligned}
& \bar{a}\left(e_{i_{n}}-s_{i_{n}+1}, e_{i_{n}}-s_{i_{n}+1}\right) \leq \bar{a}\left(e_{i_{n}}-v, e_{i_{n}}-v\right)=\bar{a}\left(e_{i_{n}}, e_{i_{n}}\right)-2 \bar{a}\left(e_{i_{n}}, v\right)+\bar{a}(v, v) \\
& \forall v \in L^{2}(\Gamma, H ; d \mu), \operatorname{dim} R(v) \leq k,
\end{aligned}
$$

and then

$$
\begin{aligned}
& \bar{a}\left(e_{i_{n}}-s_{i_{n}+1}, e_{i_{n}}-s_{i_{n}+1}\right)-\bar{a}\left(e_{i_{n}}, e_{i_{n}}\right) \\
& \quad \leq-2 \bar{a}\left(e_{i_{n}}, v\right)+\bar{a}(v, v) \quad \forall v \in L^{2}(\Gamma, H ; d \mu), \operatorname{dim} R(v) \leq k,
\end{aligned}
$$

or in other terms,

$$
\begin{aligned}
& -2 \bar{a}\left(e_{i_{n}}, s_{i_{n}+1}\right)+\bar{a}\left(s_{i_{n}+1}, s_{i_{n}+1}\right) \\
& \quad \leq-2 \bar{a}\left(e_{i_{n}}, v\right)+\bar{a}(v, v) \quad \forall v \in L^{2}(\Gamma, H ; d \mu), \operatorname{dim} R(v) \leq k .
\end{aligned}
$$

Thanks to (50) and (51), the first line tends to zero when $n$ tends to infinity, while in the second line we can pass to the limit by (52). Thus, we have

$$
2 \bar{a}(e, v) \leq \bar{a}(v, v) \quad \forall v \in L^{2}(\Gamma, H ; d \mu), \quad \operatorname{dim} \mathrm{R}(v) \leq k .
$$

Replacing in this equality $v$ by $t v$ with $t>0$, dividing by $t$, letting $t$ tend to zero, and writing the resulting inequality for $v$ and $-v$, we get

$$
\bar{a}(e, v)=0 \quad \forall v \in L^{2}(\Gamma, H ; d \mu), \quad \operatorname{dim} \mathrm{R}(v) \leq k .
$$

Taking $v=w \varphi$, with $w \in H, \varphi \in L^{2}(\Gamma ; d \mu)$, and recalling definition (2) of $\bar{a}$, we deduce

$$
\int_{\Gamma} a(e(\gamma), w ; \gamma) \varphi(\gamma) d \mu(\gamma)=0 \quad \forall z \in H, \forall \varphi \in L^{2}(\Gamma ; d \mu),
$$

and then for any $w \in H$, there exists a subset $N_{w} \in \mathcal{B}$ with $\mu\left(N_{w}\right)=0$ such that

$$
a(e(\gamma), w ; \gamma)=0 \quad \forall \gamma \in \Gamma \backslash N_{w} .
$$

The separability of $H$ implies that $N_{w}$ can be chosen independent of $w$, and then we have

$$
a(e(\gamma), w ; \gamma)=0 \quad \forall w \in H, d \mu \text {-a.e. } \gamma \in \Gamma,
$$

and therefore,

$$
e(\gamma)=0 \quad d \mu \text {-a.e. } \gamma \in \Gamma .
$$

This proves that $e$ does not depend on the subsequence in (52) and that

$$
e_{i} \rightarrow 0 \text { in } L^{2}(\Gamma, H ; d \mu)
$$

Let us now prove that in (55) the convergence is strong in $L^{2}(\Gamma, H ; d \mu)$. We use the fact that thanks to (46), we have

$$
e_{i}=-\sum_{j=1}^{i} s_{j}+e_{0} \quad \forall i \geq 1,
$$

Copyright $@$ by SIAM. Unauthorized reproduction of this article is prohibited. 
and so,

$$
\bar{a}\left(e_{i}, e_{i}\right)=-\sum_{j=1}^{i} \bar{a}\left(e_{i}, s_{j}\right)+\bar{a}\left(e_{i}, e_{0}\right) \quad \forall i \geq 1
$$

In order to estimate the right-hand side of the latest equality, we introduce, for $i, j \geq 1$, the function $z_{i, j}$ as the solution of

$$
z_{i, j} \in L^{2}\left(\Gamma, \mathrm{R}\left(s_{j}\right) ; d \mu\right), \quad \bar{a}\left(z_{i, j}, v\right)=\bar{a}\left(e_{i-1}, v\right) \quad \forall v \in L^{2}\left(\Gamma, \mathrm{R}\left(s_{j}\right) ; d \mu\right) .
$$

We have

$$
\left|\bar{a}\left(e_{i-1}, s_{j}\right)\right|=\left|\bar{a}\left(z_{i, j}, s_{j}\right)\right| \leq \bar{a}\left(z_{i, j}, z_{i, j}\right)^{\frac{1}{2}} \bar{a}\left(s_{j}, s_{j}\right)^{\frac{1}{2}} .
$$

Using (48), (46), the fact that $s_{i}$ is a solution of (45), and $\operatorname{dim} \mathrm{R}\left(s_{j}\right) \leq k$, we have

$$
\bar{a}\left(e_{i-1}, e_{i-1}\right)-\bar{a}\left(s_{i}, s_{i}\right)=\bar{a}\left(e_{i-1}-s_{i}, e_{i-1}-s_{i}\right) \leq \bar{a}\left(e_{i-1}-z_{i, j}, e_{i-1}-z_{i, j}\right) .
$$

Expanding the right-hand side and using $v=z_{i, j}$ in (57) gives

$$
\bar{a}\left(z_{i, j}, z_{i, j}\right) \leq \bar{a}\left(s_{i}, s_{i}\right),
$$

which combined with (58) provides the estimate

$$
\left|\bar{a}\left(e_{i-1}, s_{j}\right)\right| \leq \bar{a}\left(s_{i}, s_{i}\right)^{\frac{1}{2}} \bar{a}\left(s_{j}, s_{j}\right)^{\frac{1}{2}} \quad \forall i, j \geq 1 .
$$

Using the latest estimate in (56) and then the Cauchy-Schwarz inequality, we get

$$
\left\{\begin{array}{l}
\bar{a}\left(e_{i}, e_{i}\right) \leq \bar{a}\left(s_{i+1}, s_{i+1}\right)^{\frac{1}{2}} \sum_{j=1}^{i} \bar{a}\left(s_{j}, s_{j}\right)^{\frac{1}{2}}+\bar{a}\left(e_{i}, e_{0}\right) \\
\leq \bar{a}\left(s_{i+1}, s_{i+1}\right)^{\frac{1}{2}} i^{\frac{1}{2}}\left(\sum_{j=1}^{\infty} \bar{a}\left(s_{j}, s_{j}\right)\right)^{\frac{1}{2}}+\bar{a}\left(e_{i}, e_{0}\right) \quad \forall i \geq 1 .
\end{array}\right.
$$

But the criterion of comparison of two series with nonnegative terms, and the facts that (see (51))

$$
\sum_{i=1}^{\infty} \frac{1}{i}=\infty, \quad \sum_{i=1}^{\infty} \bar{a}\left(s_{i}, s_{i}\right)<\infty
$$

prove that

$$
\liminf _{i \rightarrow \infty} \bar{a}\left(s_{i+1}, s_{i+1}\right) i=\liminf _{i \rightarrow \infty} \frac{\bar{a}\left(s_{i+1}, s_{i+1}\right)}{\frac{1}{i}}=0 .
$$

Since $\bar{a}\left(e_{i}, e_{i}\right)$ is a decreasing sequence by (48), and since (55) asserts that $e_{i}$ converges weakly to zero, we can pass to the limit in (59) to deduce

$$
\begin{aligned}
& \lim _{i \rightarrow \infty} \bar{a}\left(e_{i}, e_{i}\right)=\liminf _{i \rightarrow \infty} \bar{a}\left(e_{i}, e_{i}\right) \\
& \leq \liminf _{i \rightarrow \infty}\left(\bar{a}\left(s_{i+1}, s_{i+1}\right)^{\frac{1}{2}} i^{\frac{1}{2}}\left(\sum_{j=1}^{\infty} \bar{a}\left(s_{j}, s_{j}\right)\right)^{\frac{1}{2}}+\bar{a}\left(e_{i}, e_{0}\right)\right)=0 .
\end{aligned}
$$

This proves that $e_{i}$ converges strongly to zero in $L^{2}(\Gamma, H ; d \mu)$. Since $e_{i}=u-u_{i}$ this finishes the proof of Theorem 5.3. 
Remark 5.5. In many cases the corrections $s_{i}$ decrease exponentially in the sense that

$$
\left\|s_{i}\right\|=O\left(\rho^{-i}\right) \text { as } i \rightarrow+\infty \text { for some } \rho>1,
$$

where $\|\cdot\| \|$ denotes the norm in $L^{2}(\Gamma, H ; d \mu)$. This occurs in particular for the standard POD expansion when $\Gamma$ is an open set of $\mathbb{R}^{N}, \mu$ is the Lebesgue measure, and the function $f=f(\gamma)$ is analytic with respect to $\gamma$ (see [7]). Then $\left\|s_{i}\right\|$ is a good estimator for the error $\left\|u-u_{i}\right\|$.

6. Relationship with POD and PGD methods. The "intrinsic" PGD method developed in the previous sections is a genuine extension of both the POD and PGD methods.

Indeed, to analyze the connections with the POD method, let us consider the problem studied in [11], namely,

$$
\left(P_{k}\right)^{\prime} \quad \min _{Z \in \mathbb{G}_{\leq k}} \int_{\Gamma}\left(u(\gamma)-u_{Z}(\gamma), u(\gamma)-u_{Z}(\gamma)\right)_{H} d \mu(\gamma),
$$

where $(\cdot, \cdot)_{H}$ is an inner product on $H$. In this case, a solution of $\left(P_{k}\right)^{\prime}$ is the space generated by the first $k$ eigenfunctions of the POD operator $\mathcal{P}: H \mapsto H$, which is given by (see below)

$$
\mathcal{P}(v)=\int_{\Gamma}(u(\gamma), v)_{H} u(\gamma) d \mu(\gamma) \quad \forall v \in H
$$

In the present case, due to the dependence of $a$ with respect to $\gamma$, it does not seem that the problem can be reduced to a spectral problem.

As an example, from now on we fix in this section

$$
k=1 .
$$

Then problem (17) can be written as

$$
\min _{v \in H, \varphi \in L^{2}(\Gamma ; d \mu)} \int_{\Gamma} a(u(\gamma)-\varphi(\gamma) v, u(\gamma)-\varphi(\gamma) v ; \gamma) d \mu(\gamma) .
$$

Note that problem (61) has at least a solution (see section 3 above). So, taking the derivative of the functional

$$
(v, \varphi) \in H \times L^{2}(\Gamma ; d \mu) \mapsto \int_{\Gamma} a(u(\gamma)-\varphi(\gamma) v, u(\gamma)-\varphi(\gamma) v ; \gamma) d \mu(\gamma),
$$

we deduce that if $(w, \psi) \in H \times L^{2}(\Gamma ; d \mu)$ is a solution of $(61)$, with $w \neq 0$, then

$$
\psi(\gamma)=\frac{a(u(\gamma), w ; \gamma)}{a(w, w ; \gamma)}, \quad d \mu \text {-a.e. } \gamma \in \Gamma,
$$

and $w$ is a solution of the nonlinear variational problem

$$
\int_{\Gamma} \frac{a(u(\gamma), w ; \gamma)}{a(w, w ; \gamma)} a(u(\gamma), v ; \gamma) d \mu(\gamma)=\int_{\Gamma} \frac{a(u(\gamma), w ; \gamma)^{2}}{a(w, w ; \gamma)^{2}} a(w, v ; \gamma) d \mu(\gamma) \quad \forall v \in H
$$

Note that if $w=0$,

$$
\begin{aligned}
& \int_{\Gamma} a(u(\gamma)-\varphi(\gamma) v, u(\gamma)-\varphi(\gamma) v ; \gamma) d \mu(\gamma) \\
& \quad \geq \int_{\Gamma} a(u(\gamma), u(\gamma) ; \gamma) d \mu(\gamma) \quad \forall v \in H, \forall \varphi \in L^{2}(\Gamma, d \mu) .
\end{aligned}
$$

Copyright $\odot$ by SIAM. Unauthorized reproduction of this article is prohibited. 
This implies that $u=0$ and therefore $f=0$.

If $a$ does not depend on $\gamma$, statement (63) can be written as

$$
a\left(\int_{\Gamma} a(u(\gamma), w) u(\gamma) d \mu(\gamma), v\right)=a\left(\frac{\int_{\Gamma} a(u(\gamma), w)^{2} d \mu(\gamma)}{a(w, w)} w, v\right) \quad \forall v \in H,
$$

which implies that

$$
\int_{\Gamma} a(u(\gamma), w) u(\gamma) d \mu(\gamma)=\frac{\int_{\Gamma} a(u(\gamma), w)^{2} d \mu(\gamma)}{a(w, w)} w
$$

which proves that $w$ is an eigenvector of the operator

$$
v \in H \mapsto \mathcal{P}(v)=\int_{\Gamma} a(u(\gamma), v) u(\gamma) d \mu(\gamma)
$$

for the eigenvalue

$$
\frac{\int_{\Gamma} a(u(\gamma), w)^{2} d \mu(\gamma)}{a(w, w)} .
$$

In contrast, when $a$ depends on $\gamma$ it does not seem that problem (63) corresponds to an eigenvalue problem.

To analyze the relationship of the intrinsic PGD with the standard PGD method, let us remember that this method approximates the solution $u$ of problem (8) by a series similar to that provided by the deflation algorithm introduced in section 5 , namely,

$$
u(\gamma)=\sum_{i \geq 1} \widetilde{\Phi}_{i}(\gamma) \widetilde{w}_{i}
$$

where the pair $\left(\widetilde{\Phi}_{i}, \widetilde{w}_{i}\right) \in L^{2}(\Gamma, d \mu) \times H$ is recursively obtained as a solution of the nonlinear coupled problems

(64)

$$
\left\{\begin{aligned}
\int_{\Gamma} a\left(\widetilde{\Phi}_{i}(\gamma) \widetilde{w}_{i}, \widetilde{\Phi}_{i}(\gamma) v ; \gamma\right) d \mu(\gamma) & =\int_{\Gamma}\left\langle\widetilde{f}_{i}(\gamma), \widetilde{\Phi}_{i}(\gamma) v\right\rangle d \mu(\gamma) \forall v \in H, \\
\int_{\Gamma} a\left(\widetilde{\Phi}_{i}(\gamma) \widetilde{w}_{i}, \widetilde{\Phi}^{*}(\gamma) \widetilde{w}_{i} ; \gamma\right) d \mu(\gamma) & =\int_{\Gamma}\left\langle\widetilde{f}_{i}(\gamma), \widetilde{\Phi}^{*}(\gamma) \widetilde{w}_{i}\right\rangle d \mu(\gamma) \forall \widetilde{\Phi}^{*} \in L^{2}(\Gamma, d \mu),
\end{aligned}\right.
$$

where $\widetilde{f}_{1}=f$, and $\widetilde{f}_{i}$ is defined by

$$
\int_{\Gamma}\left\langle\widetilde{f}_{i}(\gamma), v(\gamma)\right\rangle d \mu(\gamma)=\int_{\Gamma}\langle f(\gamma), v(\gamma)\rangle d \mu(\gamma)-\bar{a}\left(\widetilde{u}_{i-1}, v\right) \quad \forall v \in L^{2}(\Gamma, H ; d \mu),
$$

with

$$
\widetilde{u}_{i-1}(\gamma)=\sum_{j=1}^{i-1} \widetilde{\Phi}_{j}(\gamma) \widetilde{w}_{j} \quad \text { for } i \geq 2 .
$$

If problem (64) admits a solution such that $\widetilde{w}_{i} \neq 0$, then the second equation in (64) is equivalent to

$$
a\left(\widetilde{\Phi}_{i}(\gamma) \widetilde{w}_{i}, \widetilde{w}_{i} ; \gamma\right)=\left\langle\widetilde{f}_{i}(\gamma), \widetilde{w}_{i}\right\rangle d \mu \text {-a.e. } \gamma \in \Gamma,
$$

Copyright $@$ ( ) by SIAM. Unauthorized reproduction of this article is prohibited. 
which in turn is equivalent to

$$
\widetilde{\Phi}_{i}(\gamma)=\frac{\left\langle\widetilde{f}_{i}(\gamma), \widetilde{w}_{i}\right\rangle}{a\left(\widetilde{w}_{i}, \widetilde{w}_{i} ; \gamma\right)} d \mu \text {-a.e. } \gamma \in \Gamma \text {. }
$$

Then the first equation in (64) is equivalent to the nonlinear variational problem

$$
\widetilde{w}_{i} \in H, \int_{\Gamma} \frac{\left\langle\widetilde{f}_{i}(\gamma), \widetilde{w}_{i}\right\rangle}{a\left(\widetilde{w}_{i}, \widetilde{w}_{i} ; \gamma\right)}\left\langle\widetilde{f}_{i}(\gamma), v\right\rangle d \mu(\gamma)=\int_{\Gamma} \frac{\left\langle\widetilde{f}_{i}(\gamma), \widetilde{w}_{i}\right\rangle^{2}}{a\left(\widetilde{w}_{i}, \widetilde{w}_{i} ; \gamma\right)^{2}} a\left(\widetilde{w}_{i}, v ; \gamma\right) d \mu(\gamma) \quad \forall v \in H
$$

Note that this problem is just problem $(63)$ with $w$ replaced by $\widetilde{w}_{i}$ and $f$ replaced by $\widetilde{f}_{i}$.

Conversely, if problem (68) admits a solution, then the pair $\left(\widetilde{w}_{i}, \widetilde{\Phi}_{i}\right)$, with $\Phi$ defined by (67), is a solution of the PGD problem (64).

Consequently the sequence $\left(\Phi_{i}, w_{i}\right)$ provided by the deflation algorithm (39)-(40) is also a solution of the PGD algorithm (64)-(66), with $\tilde{f}_{i}=f_{i}$ for all $i \geq 1$. Thus, if the PGD algorithm is computed in such a way that at each step $\widetilde{\Phi}_{i} \widetilde{w}_{i}=\Phi_{i} w_{i}(=$ $s_{i}$ ), the analysis developed in section 5 proves that the sequence $\widetilde{u}_{i}$ converges in $L^{2}(\Gamma, H ; d \mu)$ to the parametric solution $u(\gamma)$ of problem (3).

However, there is the possibility that problem (64) admits several solutions and that some of these do not provide a solution of the optimization problem (45). Then the convergence properties studied in section 5 may be lost. It is then convenient to solve the PGD problem (64), ensuring that the solution does provide an optimal subspace.

The previous analysis presents some differences from preceding works on the analysis of convergence of PGD methods applied to the solution of PDEs and optimization problems. Let us describe some of them. In [2] the authors prove the convergence of the PGD for finite-dimensional linear systems $A \mathbf{x}=\mathbf{b}$ where $A \in \mathbb{R}^{N \times N}$ is an invertible high-dimensional matrix, i.e., $N=N_{1} N_{2} \cdots N_{n}$. The solution is searched as a series of rank 1 summands, belonging to $\mathbb{R}^{N}=\mathbb{R}^{N_{1}} \otimes_{a} \mathbb{R}^{N_{2}} \otimes_{a} \cdots \otimes_{a} \mathbb{R}^{N_{n}}$ (where $\otimes_{a}$ denotes the algebraic tensor product). Also, in [8] the authors prove the convergence of the PGD algorithm applied to the Laplace problem in a tensor product domain,

$$
-\Delta u=f \text { in } \Omega_{x} \times \Omega_{y},\left.\quad u\right|_{\partial \Omega_{x} \times \Omega_{y}}=0,
$$

where $\Omega_{x} \subset \mathbb{R}$ and $\Omega_{y} \subset \mathbb{R}$ are two bounded domains. The authors solve the problem on the tensor space $H_{0}^{1}\left(\Omega_{x}\right) \otimes_{a} H_{0}^{1}\left(\Omega_{y}\right)$ which is dense in $H_{0}^{1}\left(\Omega_{x} \times \Omega_{y}\right)$ for the norm of $H_{0}^{1}\left(\Omega_{x} \times \Omega_{y}\right)$. The work [9] proves the convergence of the PGD for the following optimization problem: Find $\mathbf{u} \in L^{2}\left(\Omega, H^{1}(I)\right)$ such that $\mathbf{u} \in \arg \min _{\mathbf{v} \in L^{2}\left(\Omega, H^{1}(I)\right)} \mathcal{E}(\mathbf{v})$, where $\mathcal{E}$ is a strongly convex functional, with Lipschitz gradient on bounded sets. This method can be used for high-dimensional nonlinear convex problems. Further, in [12] the authors prove the convergence of a PGD-like algorithm, where the set of rank 1 tensors in a tensor space is substituted by a closed cone $\Sigma$, to solve the following variational problem: Find $\mathbf{u} \in X$ such that $\mathbf{u} \in \arg \min _{\mathbf{v} \in X} \mathcal{E}(\mathbf{v})$, where $\mathcal{E}: X \longrightarrow \mathbb{R}$ is a convex functional defined over a reflexive Banach space $X$. Moreover, in [11] the authors prove the convergence of the PGD for elliptic PDEs in the form $A \mathbf{u}=\mathbf{f}$ where $\mathbf{u}$ and $\mathbf{f}$ belong to a Hilbert tensor space $H=\bar{H}_{1} \otimes_{a} H_{2} \otimes_{a} \cdots \otimes_{a} H_{n}{ }^{|I \cdot| \| \mid}$. Here the norm $\|\left.|\cdot|\right|^{2}=\langle\cdot, \cdot\rangle$ is given by $\langle\cdot, \cdot\rangle=\langle\cdot, \cdot\rangle_{1}\langle\cdot, \cdot\rangle_{2} \cdots\langle\cdot, \cdot\rangle_{n}$. This is a generalization of the Eckart-Young theorem.

The results reported in the present paper are a generalization of [11] when the operator $A$ depends on a parameter. 
7. Conclusion. In this paper we have introduced an iterative deflation algorithm to solve parametric symmetric elliptic equations. It is a proper generalized decomposition (PGD) algorithm, as it builds a tensorized representation of the parameterized solutions, by means of optimal subspaces that minimize the residual in the mean quadratic norm. It is intrinsic in the sense that in each deflation step the residual is minimized in the "natural" parametric norm generated by the parametric elliptic operator. It is conceptually close to the proper orthogonal decomposition (POD) with the difference that in the POD the residual is minimized with respect to a fixed mean quadratic norm. Due to this difference, spectral theory cannot be applied.

We have proved the existence of the optimal subspaces of dimension less than or equal to a fixed number, as required in each iteration of the deflation algorithm, with specific analysis for the one-dimensional case. Also, we have proved the strong convergence in the parametric elliptic norm of the deflation algorithm for quite general parametric elliptic operators.

We have further proved that the method introduced is a genuine extension of both POD and PGD methods, and that in particular it provides a theoretical analysis of the PGD method, when this method is applied in such a way that it provides the optimal subspaces: The PGD expansion is strongly convergent to the targeted solution in parametric elliptic norm.

We will next focus our research on the analysis of convergence rates of the intrinsic PGD expansion that we introduced. We will analyze whether the standard PGD provides the optimal subspaces, and compare the convergence rates with those of the POD expansion, to determine whether the use of optimal modes provides improved convergence rates. We will also work on the use of optimization techniques as an alternative way to compute the optimal modes, rather than the power iteration method that is common in PGD computations.

All the results obtained in the present paper refer to the case when $a$ is symmetric. In future work we will consider the nonsymmetric case.

\section{REFERENCES}

[1] R. Abraham, J. E. Marsden, and T. Ratiu, Manifolds, Tensor Analysis and Applications, Springer-Verlag, 2001.

[2] A. Ammar, F. Chinesta, And A. Falcó, On the convergence of a greedy rank-one update algorithm for a class of linear systems, Arch. Comput. Methods Eng., 17 (2010), pp. 473486.

[3] A. Ammar, B. Mokdad, F. Chinesta, and R. Keunings, A new family of solvers for some classes of multidimensional partial differential equations encountered in kinetic theory modeling of complex fluids, J. Non-Newtonian Fluid Mech., 139 (2006), pp. 153-176.

[4] B. I. Epureanu, L. S. TANG, And M. Paidoussis, Coherent structures and their influence on the dynamics of aeroelastic panels, Int. J. Non-Linear Mech., 39 (2004), pp. 977-991.

[5] G. Berkoz, P. Holmes, and J. L. Lumley, The proper orthogonal decomposition in the analysis of turbulent flows, Annu. Rev. Fluid Mech., 25 (1993), pp. 539-575.

[6] H. BrÉzis, Functional Analysis, Sobolev Spaces, and Partial Differential Equations, SpringerVerlag, 2010.

[7] M. Azaïez, F. Ben Belgacem, and T. Chacón Rebollo, Error bounds for POD expansions of parametrized transient temperatures, Comput. Methods Appl. Mech. Engrg., 305 (2016), pp. 501-511.

[8] C. LE BRIS, T. LELIEVRE, AND Y. MADAY, Results and questions on a nonlinear approximation approach for solving high-dimensional partial differential equations, Constr. Approx., 30 (2009), pp. 621-651.

[9] E. Cancès, T. Lelievre, And V. Ehrlacher, Convergence of a greedy algorithm for highdimensional convex nonlinear problems, Math. Models Methods Appl. Sci., 21 (2011), pp. $2433-2467$. 
[10] L. Cordier and M. Bergmann, Proper Orthogonal Decomposition: An Overview, Lecture Series 2002-04, 2003-03 and 2008-01 on Post-Processing of Experimental and Numerical Data, Von Karman Institute for Fluid Dynamics, 2008.

[11] A. FALCÓ AND A. Nouy, A proper generalized decomposition for the solution of elliptic problems in abstract form by using a functional Eckart Young approach, J. Math. Anal. Appl., 376 (2011), pp. 469-480.

[12] A. Falcó AND A. Nouy, Proper generalized decomposition for nonlinear convex problems in tensor Banach spaces, Numer. Math., 121 (2012), pp. 503-530.

[13] R. Ghanem and P. Spanos, Stochastic Finite Elements: A Spectral Approach, Springer-Verlag, 1991.

[14] G. H. Golub and Ch. F. Van Loan, Matrix Computations, 3rd ed., The Johns Hopkins University Press, 1996.

[15] H. Hotelling, Analysis of a complex of statistical variables into principal components, J. Educational Psychology, 24 (1933), pp. 417-441.

[16] P. Holmes, J. L. Lumley, And G. Berkooz, Coherent Structures, Dynamical Systems and Symmetry, Cambridge Monogr. Mech., Cambridge University Press, 1996.

[17] I. T. Jollife, Principal Component Analysis, Springer, 1986.

[18] M. Kahlbacher and S. VolKwein, Galerkin proper orthogonal decomposition methods for parameter depending elliptic systems, Discuss. Math. Differ. Incl. Control Optim., 27 (2007), pp. $95-117$.

[19] K. Kunisch AND S. Volkwein, Galerkin proper orthogonal decomposition methods for parabolic problems, Numer. Math., 90 (2001), pp. 117-148.

[20] K. Kunisch and S. Volkwein, Optimal snapshot location for computing POD basis functions, M2AN Math. Model. Numer. Anal., 44 (2010), pp. 509-529.

[21] O. Lass and S. Volkwein, Adaptive POD basis computation for parametrized nonlinear systems using optimal snapshot location, Comput. Optim. Appl., 58 (2014), pp. 645-677.

[22] C. LE BRIS, T. LeliÈVRE, AND Y. MADAY, Results and questions on a nonlinear approximation approach for solving high-dimensional partial differential equations, Constr. Approx., 30 (2009), pp. 621-651.

[23] M. M. Lò̀ve, Probability Theory, Van Nostrand, 1988.

[24] Y. MADAY, Reduced basis method for the rapid and reliable solution of partial differential equations, International Congress of Mathematicians, Vol. III, Eur. Math. Soc., Zürich, 2006, pp. 1255-1270.

[25] J. Margalef-Roig and E. Outerelo Dominguez, Differential Topology, North-Holland, 1992.

[26] M. Muller, On the POD Method. An Abstract Investigation with Applications to ReducedOrder Modeling and Suboptimal Control, Ph.D. Thesis, Georg-August Universität, 2008.

[27] A. NouY, A generalized spectral decomposition technique to solve a class of linear stochastic partial differential equations, Comput. Methods Appl. Mech. Engrg., 196 (2007), pp. 45214537.

[28] A. Nouy and O. P. Le Mâ̂TRE, Generalized spectral decomposition method for stochastic nonlinear problems, J. Comput. Phys., 228 (2009), pp. 202-235.

[29] J. Chen AND V. Shapiro, Optimization of continuous heterogeneous models, in Heterogeneous Objects Modelling and Applications, Lecture Notes in Comput. Sci. 4889, A. Pasko, V. Adzhiev, and P. Comninos, eds., Springer-Verlag, 2008, pp. 193-213.

[30] K. Pearson, On lines and planes of closest fit system of points in space, Philos. Mag. (6), 2 (1901), pp. 559-572.

[31] A. Quarteroni And G. Rozza, Reduced Order Methods for Modeling and Computational Reduction, MS\&A. Model. Simul. Appl. 9, Springer, 2014.

[32] W. H. A. Schilders, H. A. van der Vorst, and J. Rommes, eds. Model Order Reduction: Theory, Research Aspects and Applications, Math. Ind. 13, Eur. Consort. Math. Ind. (Berl.), Springer-Verlag, 2008.

[33] S. Torquato, Optimal design of heterogeneous materials, Annu. Rev. Mater. Res., 40 (2010), pp. 101-129, https://doi.org/10.1146/annurev-matsci-070909-104517.

[34] S. Volkwein, Proper Orthogonal Decomposition: Theory and Reduced-Order Modelling, Lecture Notes, Department of Mathematics and Statistics, University of Konstanz, 2013.

Copyright (c) by SIAM. Unauthorized reproduction of this article is prohibited. 